\title{
Ensemble Pulsar Time Study by Pulsar Timing Observations
}

\author{
Tinggao Yang \& Guangren Ni \\ National Time Service Center, Chinese Academy of Sciences, P.O. Box \\ 18, Lintong, Shaanxi, China
}

\begin{abstract}
Long term timing of multiple millisecond pulsars can contribute to the study of an ensemble pulsar time scale PTens. A wavelet decomposition algorithm (WDA) was applied to define a PTens using the available millisecond pulsar timing data. The PTens obtained from WDA is more stable than those resulting from other algorithms. The Chinese $50 \mathrm{~m}$ radio telescope is specially designed for PTens study and detection of gravitational wave background via millisecond pulsars timing observations. A scheme for multiple millisecond pulsar timing and ensemble pulsar time study is discussed in some detail.
\end{abstract}

\section{Introduction}

Long term timing data analysis of some millisecond pulsars, for example PSRs $\mathrm{B} 1937+21$ and B1855+09, showed that the stability of rotation of such pulsars rivals that of atomic clocks (Kaspi et al. 1994). In order to find a stable pulsar time scale, an ensemble pulsar time scale PTens can be constructed using individual pulsar time scale PTs (Petit \& Tavella 1996). As many new millisecond pulsars are discovered, it is possible to monitor almost simultaneously several millisecond pulsars using one radio telescope. A timing array could lead to the study of a PTens for long time scales and detection of a gravitational wave background (GWB; Foster \& Backer 1990). A Chinese $50 \mathrm{~m}$ radio telescope specially designed for timing millisecond pulsars will take part in studying PTens and detecting the GWB.

\section{Algorithm for PTens}

In addition to the intrinsic noise of the rotation of millisecond pulsars and errors of atomic time scale AT used as a reference, several other sources of noise could affect timing data. Chief among them are interstellar propagation effects, uncertainties in the ephemeris of the solar system, and gravitational waves. For convenience, one assumes that all sources of timing noise except AT are independent for different pulsars and then one averages them by defining PTens as a weighted average of the individual PT from all the available pulsars. Following this algorithm, Petit \& Tavella (1996) calculated an ensemble pulsar time scale using data from PSRs B1937+21 and B1855+09. We have applied the wavelet decomposition algorithm (WDA) to define an ensemble pulsar time scale using 
the same data from these two pulsars. The method of using wavelet analysis to calculate time scales is different from that of uniform weighting in the time domain. WDA can decompose signals' subsection components in the range of various frequencies and then pick them up again to weight in subdomains. WDA considers, analyzes and solves the stability of subsection frequency in the range of various frequencies. Our results indicated that the time scale calculated by WDA is more stable.

\section{Pulsar Timing Program with the $50 \mathrm{~m}$ Radio Telescope}

Construction of a $50 \mathrm{~m}$ radio telescope is underway and will be completed in two years. The telescope will be installed at Miyun station of the National Astronomical Observatory in China. Jodrell Bank Observatory is developing a Coherent On-line Baseband Recorder for Astronomy (COBRA), which will perform the pulsar data processing on-line in software. The $50 \mathrm{~m}$ telescope will be equipped with a COBRA-like system. Efficiency of the antenna is about 0.5 for a center frequency of $1665 \mathrm{MHz}$, the system temperature is about $30 \mathrm{~K}$, and bandwidth is $300 \mathrm{MHz}$. More than 10 millisecond pulsars will be monitored regularly. Integration time is $60 \mathrm{~min}$ or more for each daily observation of each pulsar. According to the parameters of millisecond pulsars available today, more than 30 millisecond pulsars can be observed by the Chinese $50 \mathrm{~m}$ telescope. Eventually, about 10 suitable objects with high flux density, sharp pulse, and short rotational period will be monitored. When more suitable millisecond pulsars are found, they can be added to our program to insure that the spatial distribution of the monitored pulsar population can be more uniform. The regular timing observations should be made for the target pulsars to get timing data spanning many years. We hope that some insight about the behavior of long term stability of pulsar time scales may be learned. An ensemble pulsar time PTens can be studied by a suitable algorithm. When we define a pulsar time scale PT, rotational and astrometric parameters of pulsar are determined by an adjustment using AT as a reference time scale. An adjusted function absorbs some of the instability of AT. Consequently this necessary adjustment introduces a dependence of $\mathrm{PT}$ on $\mathrm{AT}$ and reduces the possible use of the stability of PT. The observations of a number of millisecond pulsars distributed across the sky lead to the possibility of timing several pulsars against each other. Now several long term timing projects are underway to monitor these millisecond pulsars. Timing data from different sites may be linked together by common target observations.

\section{References}

Foster, R. S., \& Backer, D. C. 1990, ApJ, 361, 300

Kaspi, V. M., Taylor, J. H., \& Ryba, M. F. 1994, ApJ, 428, 713

Petit, G., \& Tavella, P. 1996, A\&A, 308, 290 